\title{
Silent hydronephrosis/pyonephrosis due to upper urinary tract calculi in spinal cord injury patients
}

\author{
S Vaidyanathan ${ }^{*, 1}$, G Singh ${ }^{1}$, BM Soni ${ }^{1}$, P Hughes $^{2}$, JWH Watt ${ }^{1}, \mathrm{~S}$ Dundas ${ }^{3}$, P Sett ${ }^{1}$ and KF Parsons ${ }^{4}$ \\ ${ }^{1}$ Regional Spinal Injuries Centre, District General Hospital, Southport, Merseyside, UK; ${ }^{2}$ Department of Radiology, \\ District General Hospital, Southport, Merseyside, UK, ${ }^{3}$ Department of Cellular Pathology, District General Hospital, \\ Southport, Merseyside, UK; ${ }^{4}$ Department of Urology, Royal Liverpool University Hospital, Liverpool, UK
}

Study design: A study of four patients with spinal cord injury (SCI) in whom a diagnosis of hydronephrosis or pyonephrosis was delayed since these patients did not manifest the traditional signs and symptoms.

Objectives: To learn from these cases as to what steps should be taken to prevent any delay in the diagnosis and treatment of hydronephrosis/pyonephrosis in SCI patients.

Setting: Regional Spinal Injuries Centre, Southport, UK.

Methods: A retrospective review of cases of hydronephrosis or pyonephrosis due to renal/ ureteric calculus in SCI patients between 1994 and 1999, in whom there was a delay in diagnosis.

Results: A T-5 paraplegic patient had two episodes of urinary tract infection (UTI) which were successfully treated with antibiotics. When he developed UTI again, an intravenous urography (IVU) was performed. The IVU revealed a non-visualised kidney and a renal pelvic calculus. In a T-6 paraplegic patient, the classical symptom of flank pain was absent, and the symptoms of sweating and increased spasms were attributed to a syrinx. A routine IVU showed non-visualisation of the left kidney with a stone impacted in the pelviureteric junction. In two tetraplegic patients, an obstructed kidney became infected, and there was a delay in the diagnosis of pyonephrosis. The clinician's attention was focused on a co-existent, serious, infective pathology elsewhere. The primary focus of sepsis was chest infection in one patient and a deep pressure sore in the other. The former patient succumbed to chest infection and autopsy revealed pyonephrosis with an abscess between the left kidney and left hemidiaphragm and xanthogranulomatous inflammation of perinephric fatty tissue. In the latter patient, an abdominal X-ray did not reveal any calculus but computerised axial tomography showed the presence of renal and ureteric calculi.

Conclusions: The symptoms of hydronephrosis may be bizarre and non-specific in SCI patients. The symptoms include feeling unwell, abdominal discomfort, increased spasms, and autonomic dysreflexia. Physicians should be aware of the serious import of these symptoms in SCI patients.

Spinal Cord (2000) 38, 661-668

Keywords: hydronephrosis; pyonephrosis; spinal cord injury; kidney-calculus; urinary tract infection

\section{Introduction}

The clinical presentation of acute abdomen in spinal cord injury (SCI) patients varies depending upon (i) the level of injury; (ii) completeness of the spinal cord lesion; and (iii) duration of injury. Diagnoses are often so delayed that approximately $10 \%$ of these patients die of acute abdominal problems. ${ }^{1}$ Strauther and associates $^{2}$ reviewed 26 patients with spinal cord injury who later developed appendicitis over a 5-year period. The 30 -day mortality rate was $4 \%$. These

*Correspondence: S Vaidyanathan, Regional Spinal Injuries Centre, District General Hospital, Town Lane, Southport, Merseyside PR8 6PN, UK

authors concluded that the diagnosis of appendicitis was often made late in the SCI patients and complications were common. Computed tomography appeared to be an excellent diagnostic modality for diagnosis of perforated appendix with abscess. ${ }^{1}$ Bar-On and $\mathrm{Ohry}^{3}$ reviewed the occurrence of acute abdomen in 1300 patients with SCI, over 14-year period of 1978-1991. The classical signs of an 'acute abdomen' were missing in the patients with neurological levels above T-6.

Similar to the cases of acute abdomen and acute appendicitis in SCI patients, the diagnosis of an acute illness of the urinary tract may be delayed in spinal 
cord injury patients. Intra-peritoneal rupture of the bladder due to blocked catheter proved fatal in a female paraplegic, as the classic signs were absent. ${ }^{4}$ Bilateral pyelonephritis resulted in a fatal outcome in another SCI patient, as the seriousness of the illness was not recognised promptly. ${ }^{5}$ Urinary lithiasis is a common cause of morbidity in SCI patients. ${ }^{6}$ Diagnosis of hydronephrosis or pyonephrosis due to urinary calculi could be delayed in SCI patients who have a complete lesion above T-6, since these patients do not manifest the traditional signs and symptoms of renal colic. Any delay in the diagnosis of obstructive uropathy may have serious consequences. There could be irreversible loss of renal function. We present four SCI patients in whom a diagnosis of hydronephrosis or pyonephrosis due to upper urinary calculus was made belatedly since these patients did not manifest the traditional signs and symptoms.
Case reports

The clinical details of the four SCI patients are summarised in Table 1. An intravenous urography (IVU) performed during a routine follow-up in these four patients showed excretion of contrast by both kidneys with bilateral undilated pelvicalyceal systems. However, during the next 17 to 63 months, all the four patients developed hydronephrosis due to a stone impacted in the pelviureteric junction, or in the proximal ureter.

Patient A had a routine assessment in August 1994. IVU showed bilateral lower pole renal calculi and normal kidneys and ureters (Figure 1A). $\mathrm{He}$ was admitted with urinary tract infection (UTI) in 1995. Xray KUB showed a calculus at the lower pole of the left kidney. He recovered with antibiotics and was discharged home. He developed bilateral epididymoorchitis in 1997, and recovered with antibiotics. He

Table 1 Summary of the clinical details of the four SCI patients in whom a diagnosis of hydronephrosis or pyonephrosis was made belatedly

\begin{tabular}{|c|c|c|c|c|}
\hline Patient identification & $A$ & $B$ & $C$ & $D$ \\
\hline Sex & Male & Male & Male & Male \\
\hline Year of birth & 1975 & 1948 & 1956 & 1971 \\
\hline $\begin{array}{l}\text { Year when spinal cord } \\
\text { injury was sustained }\end{array}$ & 1992 & 1969 & 1977 & 1991 \\
\hline $\begin{array}{l}\text { Level of spinal cord } \\
\text { injury }\end{array}$ & $\mathrm{T}-5$ & $\mathrm{~T}-6$ & C-4 & C-6 \\
\hline Frankel Grade & A & A & A & A \\
\hline $\begin{array}{l}\text { Current method of } \\
\text { bladder drainage }\end{array}$ & $\begin{array}{l}\text { Indwelling urethral } \\
\text { catheter }\end{array}$ & $\begin{array}{l}\text { Intermittent } \\
\text { catheterisation }\end{array}$ & $\begin{array}{l}\text { Indwelling urethral } \\
\text { catheter }\end{array}$ & $\begin{array}{l}\text { Suprapubic catheter } \\
\text { drainage }\end{array}$ \\
\hline $\begin{array}{l}\text { Past medical history } \\
\text { pertaining to the } \\
\text { urinary tract }\end{array}$ & $\begin{array}{l}\text { Electrohydraulic } \\
\text { lithotripsy of bladder } \\
\text { calculus on } 24 \text { May } \\
1994 \text {. }\end{array}$ & $\begin{array}{l}\text { A } 5 \mathrm{~mm} \text { radio opaque } \\
\text { calculus in the mid- } \\
\text { pole of left kidney }\end{array}$ & $\begin{array}{l}\text { Small radio opaque } \\
\text { shadow in the left } \\
\text { renal region - } 1986 \text {. } \\
\text { Multiple bladder } \\
\text { stones-October } 1997\end{array}$ & $\begin{array}{l}1.5 \times 1 \mathrm{~cm} \text { calculus in } \\
\text { the left renal pelvis } \\
\text { in } 1994 . \text { Left ureteric } \\
\text { stenting was done on } \\
25 / 10 / 94 \text {. } \\
\text { Extracorporeal } \\
\text { shockwave } \\
\text { lithotripsy ( } 2191 \\
\text { shock waves) in } \\
\text { October } 1994\end{array}$ \\
\hline $\begin{array}{l}\text { Date of last } \\
\text { intravenous } \\
\text { urography (IVU) in } \\
\text { the spinal unit which } \\
\text { showed normal } \\
\text { kidneys }\end{array}$ & $\begin{array}{l}\text { August 1994: Bilateral } \\
\text { lower pole renal } \\
\text { calculi. Both kidneys } \\
\text { excrete contrast. } \\
\text { Normal kidneys, } \\
\text { pelvicalyceal systems } \\
\text { and ureters. }\end{array}$ & $\begin{array}{l}9 \text { September 1994: A 5- } \\
\text { mm opaque calculus } \\
\text { was present in the } \\
\text { mid-pole of left } \\
\text { kidney. }\end{array}$ & 16 January 1996 & 13 March 1997 \\
\hline $\begin{array}{l}\text { Date when unilateral } \\
\text { hydronephrosis or } \\
\text { non-visualisation of } \\
\text { kidney was detected } \\
\text { during IVU }\end{array}$ & 29 November 1999 & 20 June 1997 & 17 June 1997 & 12 May 1999 \\
\hline
\end{tabular}


A

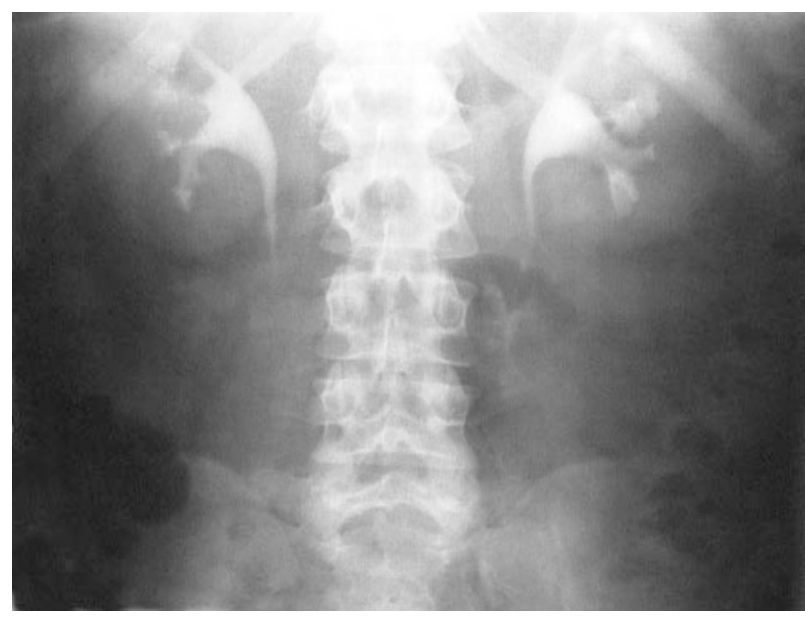

B

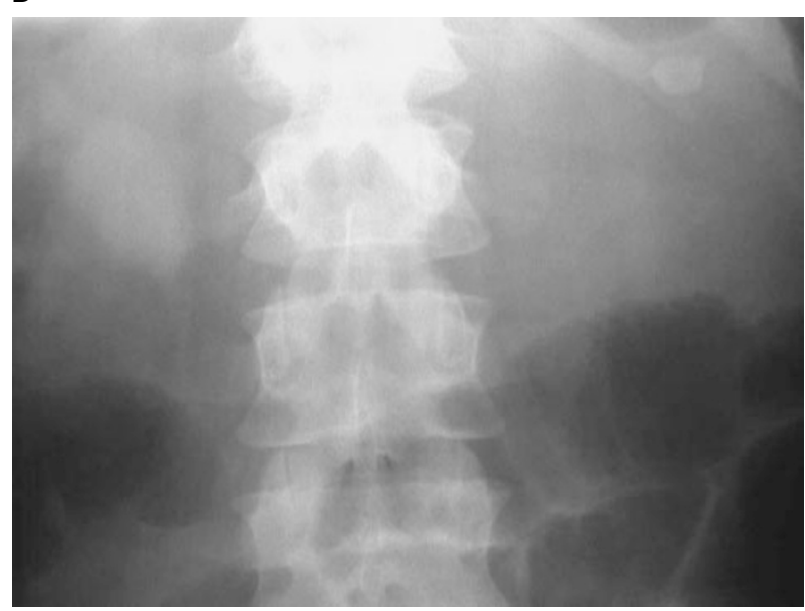

C

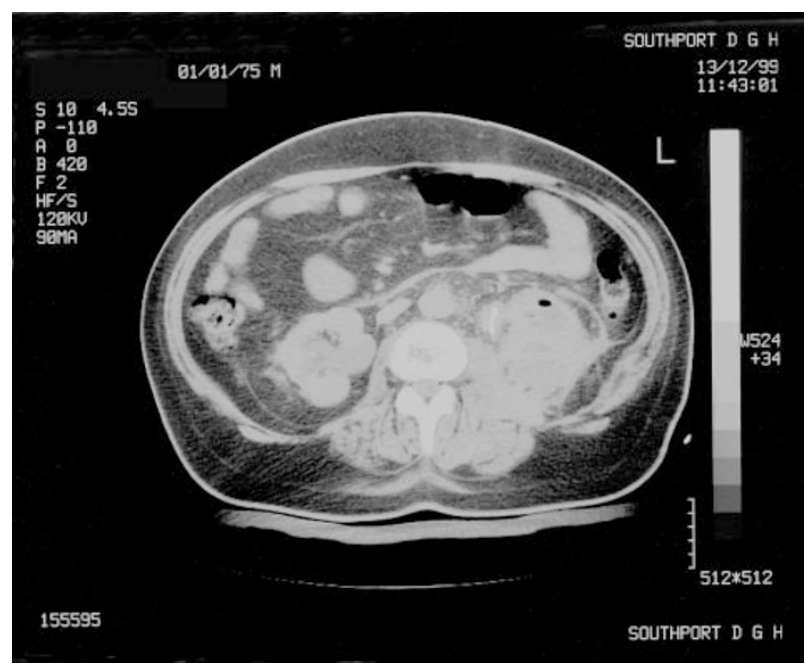

Figure 1 (A) IVU (15.8.94) shows normal kidneys, pelvicalyceal systems and ureters. (B) IVU (29.11.99) shows left renal calculi and non-functional kidney at $3 \mathrm{~h}$; normal right kidney. (C) CT of abdomen shows intra-renal and peri-renal haematoma on the left side following nephrostomy. There is a mild psoas haematoma and thickening of the perirenal fascia became asymptomatic, and a follow-up assessment of the upper urinary tract was not performed due to various reasons. He developed UTI in 1999. Temperature persisted despite antibiotics. Therefore an IVU was performed. The IVU revealed non-visualisation of the left kidney (Figure 1B). Ultrasound of the kidney showed hydronephrosis with two calculi at the left renal pelvis. Retrograde pyelography showed dilated pelvis and calyces. Pus was drained through the ureteric catheter. A five Fr. ureteric stent was inserted. Since the patient continued to have temperature of $39^{\circ} \mathrm{C}$ even after providing internal drainage by means of a stent, percutaneous nephrostomy was performed. Following percutaneous nephrostomy, his condition improved. Extracorporeal shock wave lithotripsy of left renal pelvic calculi was performed. A total of 2007 and 1531 shock waves were delivered to the left kidney stone in two separate sessions while the patient was lying in a lateral position. Subsequently, X-ray KUB revealed no radio opaque calculus in the left kidney and ureter. This patient received a course of teicoplanin as the urine specimen grew methicillin-resistant Staphylococcus aureus.

Patient B had a routine assessment in 1994. The IVU showed a $5 \mathrm{~mm}$ radio opaque calculus in the midpole of left kidney, otherwise normal kidneys, pelvicalyceal systems, and ureters (Figure 2A). In 1995, this patient presented with sweating and increased spasms. Clinical examination revealed neurological deficit in the left chest wall and left arm in the form of numbness and some weakness. MRI showed a syrinx starting at the level of C-2 and extending down the cervical and thoracic spinal cord to the lower border of T-11 (Figure 2B). The possibility of any co-existent lesion in the urinary tract was not considered. Due to numerous reasons, bi-annual evaluation of the urinary tract was missed in this case. Subsequently, a routine IVU showed nonvisualisation of the left kidney with a stone impacted in the pelviureteric junction (Figure 2C).

Ultrasound revealed a markedly hydronephrotic kidney. MAG-3 renogram performed in 1997 revealed single functioning right kidney showing normal uptake and excretion. There was no appreciable function in the hydronephrotic kidney. He was prescribed lowdose, prophylactic antibacterial therapy. He has been asymptomatic and therefore, no intervention is planned on the hydronephrotic kidney. The syrinx also remains under observation.

Patient $\mathrm{C}$ presented with sepsis and respiratory failure. Tracheostomy was performed, and he was ventilated. He recovered from this episode of chest infection and was weaned off the ventilator. Tracheal aspirate yielded a heavy growth of Pseudomonas aeruginosa. An IVU and subsequently, a CT of abdomen were performed. These imaging studies revealed normal right kidney and ureter. An opaque calculus was projected over the left renal pelvis. The left kidney was hydronephrotic (Figure 3A,B). 
A

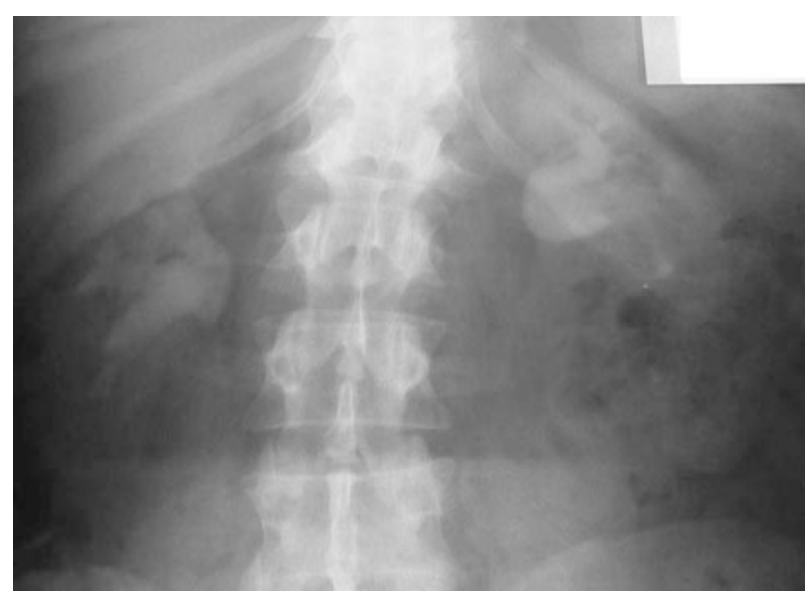

B

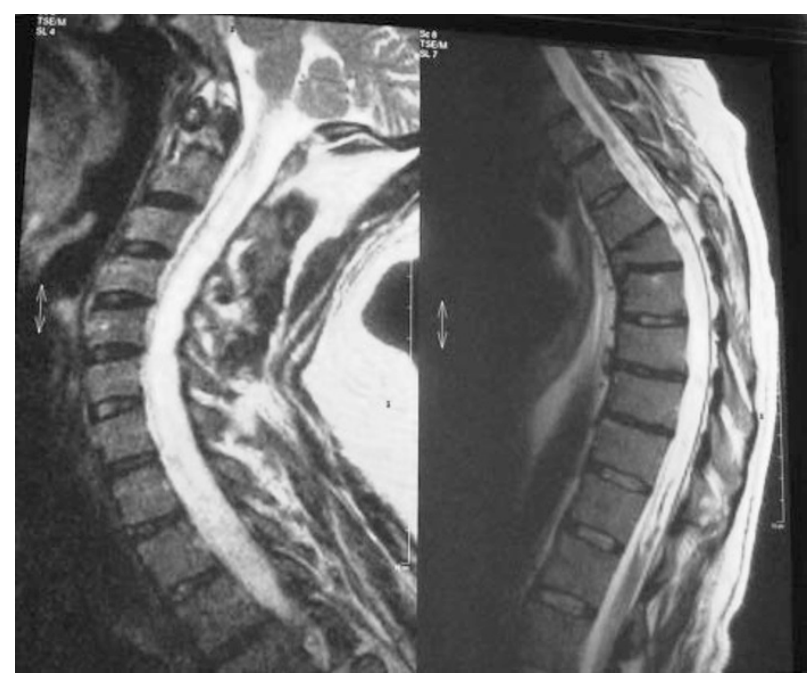

C

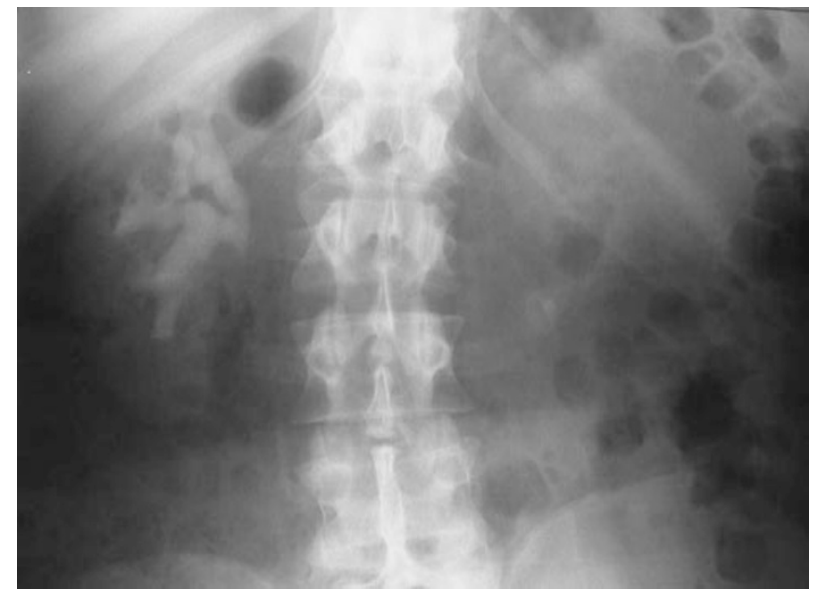

Figure 2 (A) IVU (9.9.94) shows normal kidneys, pelvicalyceal systems, and ureters. (B) MRI of spine (M353/95) shows wedge compression fracture of $\mathrm{T}-6$. There is a syrinx starting at the level of C-2 extending down the cervical and thoracic spinal cord to a level of the lower border of T-11. (C) IVU (20.6.97) shows a calculus in the left kidney; no function is demonstrated on the left kidney in the 40-min film; mild dilatation of right pelvicalyceal system
Microbiology of urine showed Pseudomonas aeruginosa. Four months later, he was admitted with severe chest infection. A chest X-ray showed long-standing atelectasis of the lower lobe of left lung with obscuration of the left hemi-diaphragm (Figure 3C). Therefore, the possibility of a co-existent, subdiaphragmatic pathology was not considered. His general condition deteriorated rapidly. He did not wish to be ventilated. He succumbed to progressive respiratory failure and sepsis. Post mortem revealed an abscess between the left kidney and left hemidiaphragm. The renal pelvis was distended with green pus. Histology showed chronic pyelonephritis of the left kidney associated with perinephric and xanthogranulomatous inflammation of perinephric fatty tissue (Figure 3D).

Patient D presented with abdominal discomfort. Gastroscopy showed oesophagitis. An IVU was performed and this showed three calcified fragments in the lower pole of the right kidney but no evidence of ureteric obstruction (Figure 4A). He continued to feel unwell. Treatment measures were focused on the infected pressure sore in the left groin, which was rightly considered as the focus of sepsis. Methicillin-resistant Staphylococcus aureus (MRSA) was isolated from the groin wound and from urine. Computerised tomography (CT) of pelvis showed changes of osteomyelitis of the left superior pubic ramus with an abscess cavity containing fluid and gas pointing anteriorly to the skin at the left groin (Figure 4B). The abscess was closely related to adductor muscles of the thigh and was tracking along the medial aspect of femoral shaft. He received multiple courses of antibiotics and there was transient improvement in his general condition. However, he started having temperature and sweating again. Therefore, an IVU was performed. This IVU revealed a non-functioning right kidney (Figure 4C). Ultrasound scan of the right kidney showed moderate hydronephrosis. Percutaneous nephrostomy was performed followed by antegrade stenting to provide internal drainage to the obstructed kidney. Microbiology of pus from the kidney showed no growth. CT of kidneys revealed a calculus in the renal pelvis and another stone partially obstructing the proximal right ureter (Figure 4D). A follow-up MAG-3 renogram showed only $3 \%$ function in the right kidney. Therefore, right nephroureterectomy was planned. As regards the pressure sore, he developed subluxation of the left hip, and required Girdlestone operation.

\section{Discussion}

It is important that SCI patients undergo assessment of the urinary tract at periodic intervals. Failure to carry out regular evaluation of the urinary tract in SCI patients may have serious consequences. These four cases illustrate the need for frequent assessment of the urinary tract in SCI patients. 
A

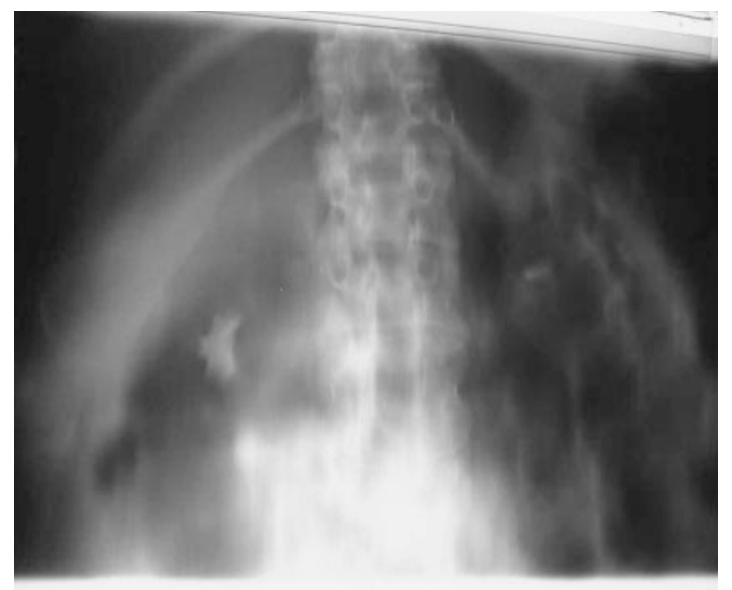

B

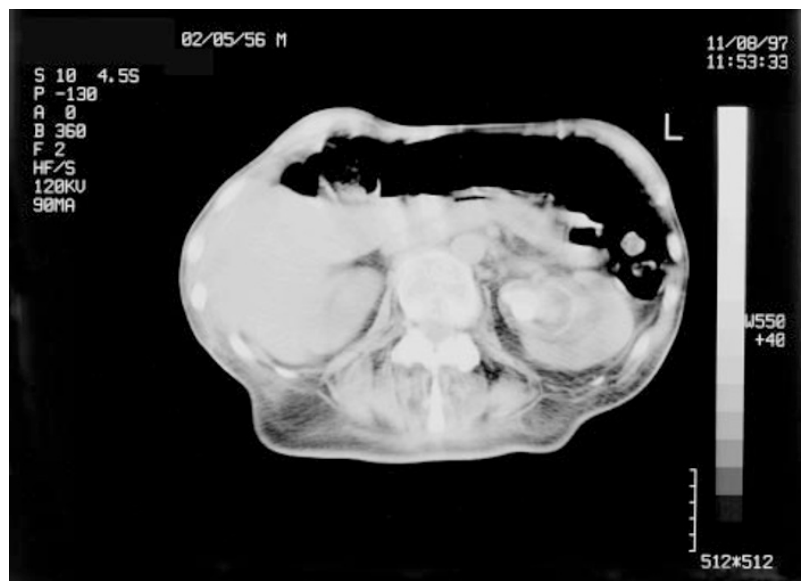

C

\section{D}
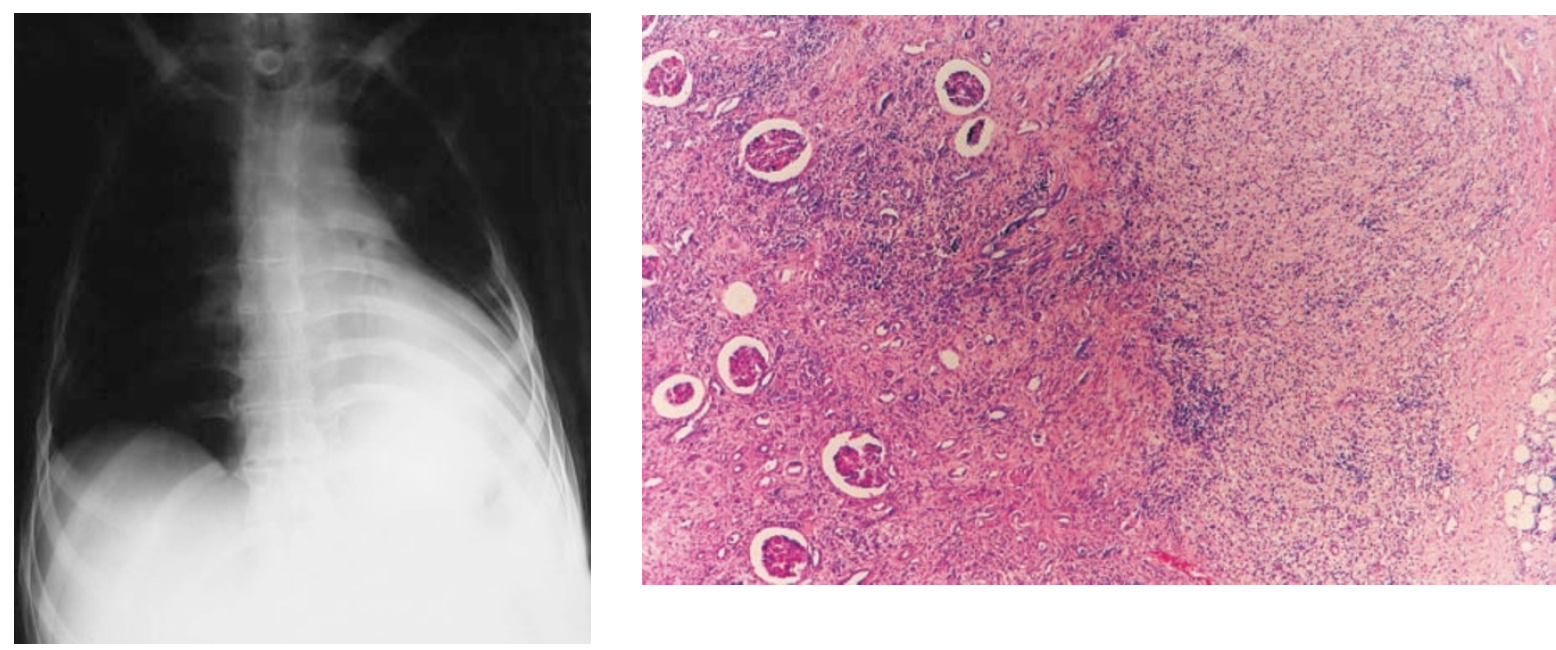

Figure 3 (A) IVU (17.6.97) - cross kidney tomogram shows opaque calculus projected over the left renal pelvis. The right kidney and ureter are normal. There is left hydronephrosis; a large, lucent filling defect is seen in the pelvicalyceal system, which represents matrix calculi. (B) CT (11.8.97) shows opaque calculus impacted at pelviureteric junction of left kidney with hydronephrosis and calcific debris within the renal pelvis. (C) Chest X-ray (30.12.97) shows long-standing collapse/consolidation of the left lower lobe with obscuration of the left hemi-diaphragm. (D) Post mortem histology of left kidney showing chronic pyelonephritis associated with xanthgranulomatous inflammation of perinephric fatty tissue. $\mathrm{H} \& \mathrm{E} \times 10$

Waites and associates ${ }^{7}$ compared the effective renal plasma flow (ERPF) in 59 patients with SCI who had not been examined for 3-15 years (years post-injury: $7-17$ ) with 101 controls (years post-injury: $6-17$ ) who were examined on an annual basis. Mean initial and follow-up ERPF values adjusted for increasing age were not significantly different for subjects and controls, nor was there significant change within individuals over time. These authors concluded that although serial examination of the urinary tract following SCI is important, after the first few years, it would be acceptable to lengthen the periods between examinations.

In our centre, we aim to review the SCI patients once in 12 months. However, we are able to assess most of the SCI patients only every 18-24 months.
In some patients, the interval between successive evaluations is longer than 24 months due to various reasons.

Canupp and associates ${ }^{8}$ listed the following reasons for non-compliance with annual follow-up evaluations in persons with SCI: (1) cost; (2) distance; (3) transportation; (4) belief that follow-up was not necessary; (5) time; and (6) availability of good local doctor. In our experience, ignorance of the need for regular follow-up assessment of the urinary tract on the part of the patient, carer and health professionals and inadequate resources are the main reasons for a SCI patient not undergoing annual evaluation. At times, there is a shortage of beds in the spinal unit, especially for follow-up assessment of SCI patients. 
A

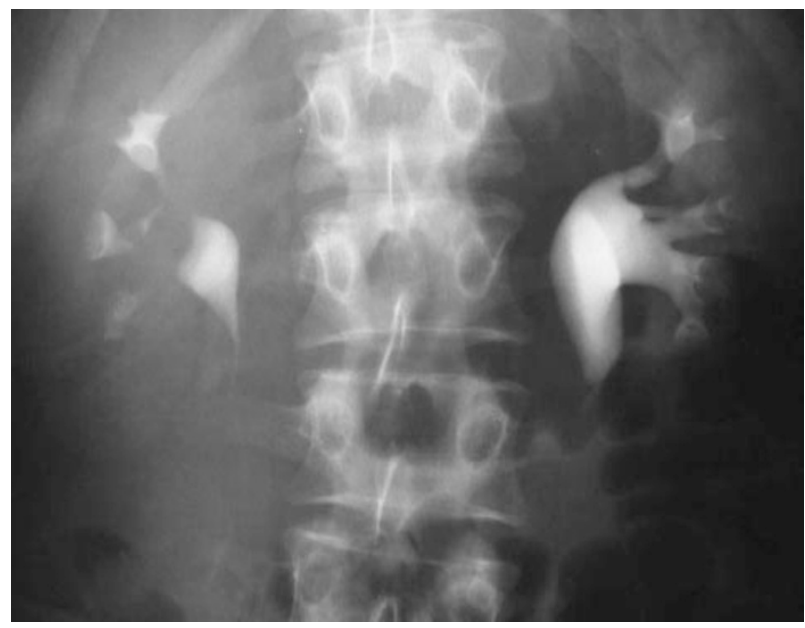

B(i)

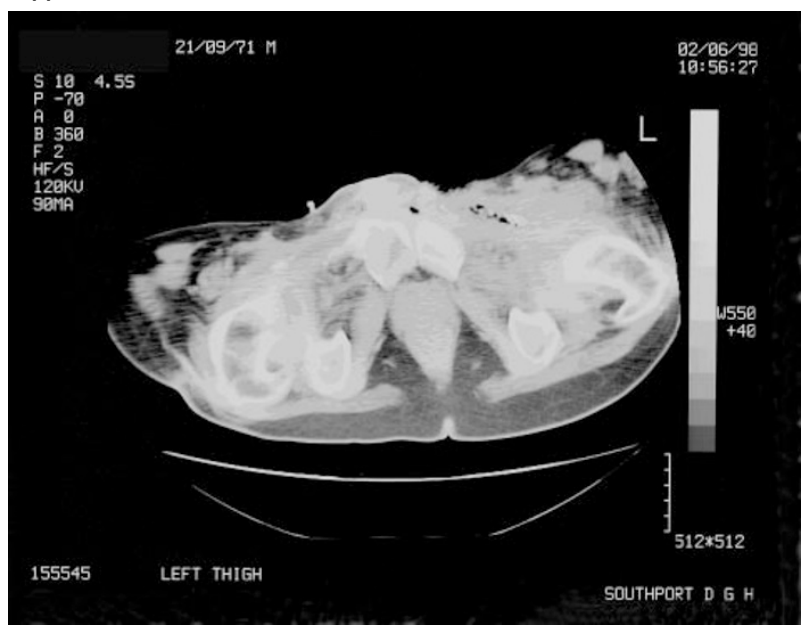

B(ii)

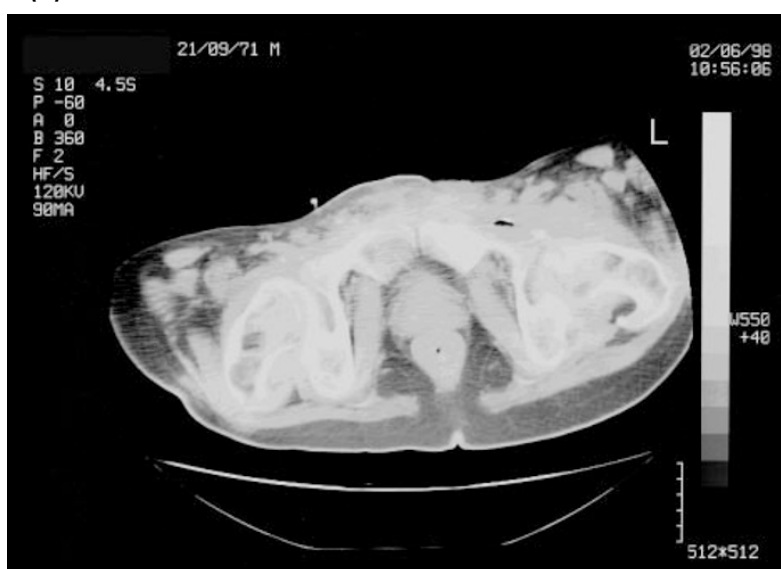

155545

LEFT THIGH
C

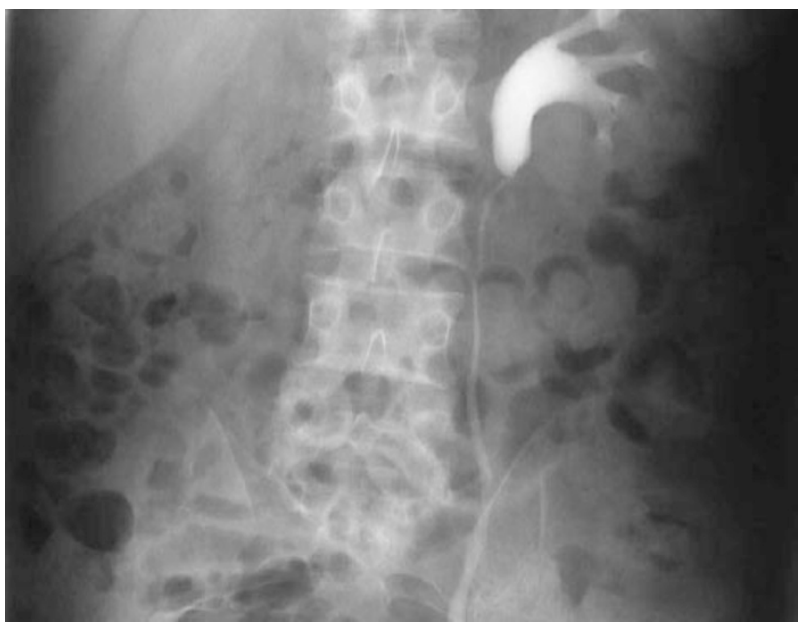

D(i)

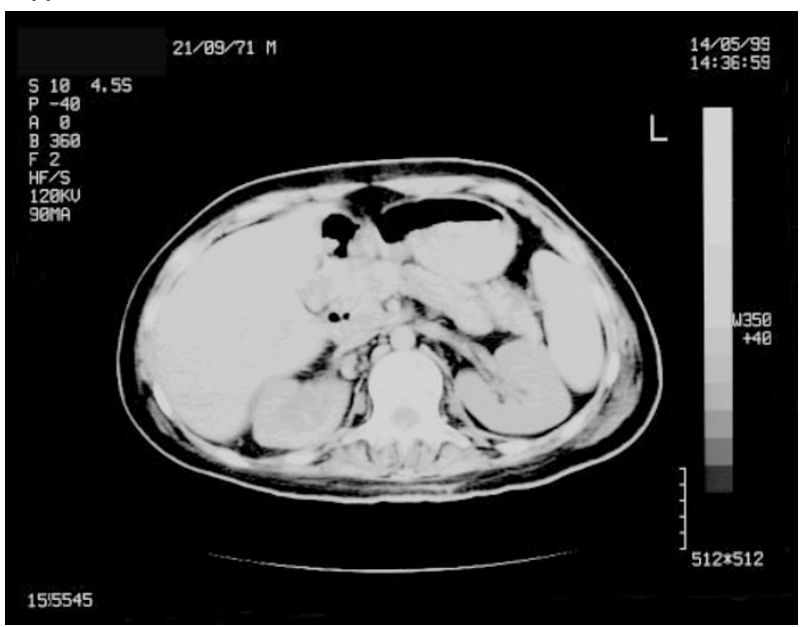

D(ii)

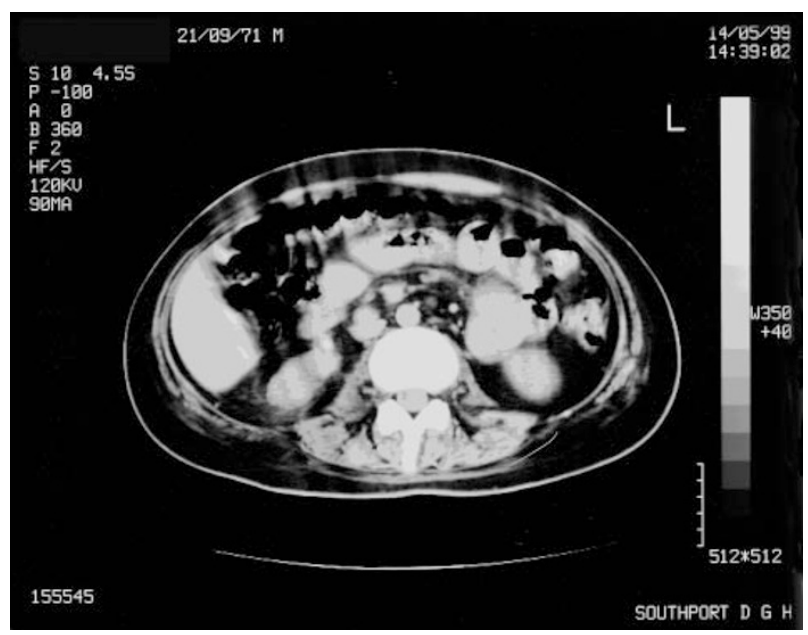

Figure 4 (A) IVU (13.3.97) shows right lower pole calculi; normal right calyceal system, and baggy extrarenal pelvis on the left. (B) CT (C-900/98) of pelvis shows changes of osteomyelitis of the left superior pubic ramus with an abscess cavity containing fluid and gas pointing anteriorly to the skin at the left groin. (C) IVU (12.5.99) shows no radio opaque calculi. Normal left kidney, and non-functioning right kidney. (D) CT (C-797/99) shows a calculus in the right renal pelvis and a second calculus partially obstructing the proximal right ureter 
The lessons learnt from the case histories of these four SCI patients who developed hydronephrosis/ pyonephrosis are summarised below.

SCI patients with UTI require radiological investigations to exclude obstructive uropathy

If a SCI patient develops UTI, in addition to antibacterial therapy, diagnostic tests must be performed to look for obstructive uropathy in the upper and lower urinary tract. Urinary tract obstruction, eg renal pelvic stone causing hydronephrosis, often manifests itself as UTI in a SCI patient. As some SCI patients tend to have constipation or gaseous distension of intestines, a faintly opaque renal or ureteric stone may be obscured in an abdominal X-ray by bowel gas or faecal matter. In these cases, unenhanced computerised tomography is of great value to detect upper urinary calculus. Unenhanced computerised axial tomography (CT) of kidneys was found to be superior to plain renal tomography to detect renal calculus and to find out the precise location of the calcified shadow within the kidney. The imaging studies should be carried out expeditiously in SCI patients; otherwise complications due to delay in diagnosis of upper urinary tract calculus may supervene. ${ }^{9}$ This was exemplified by Patient $\mathrm{D}$, in whom an X-ray of kidneys failed to reveal any urinary calculus, but computerised tomography of kidneys showed a calculus in the right renal pelvis and a second calculus partially obstructing the proximal right ureter.

Hammond and associates ${ }^{10}$ recommended diagnostic ultrasound upon diagnosis of pyelonephritis in SCI patients. The potential benefits of early imaging seem to far outweigh the negligible risk and expense. Rarely, ultrasound examination of the kidneys may be technically difficult in some SCI patients, especially those with scoliosis. We use IVU as a first-line investigation to exclude obstructive uropathy in these cases, and then proceed to ultrasound if the IVU shows a hydronephrotic or non-functioning kidney.

\section{SCI patients with recurrent UTI are at high risk for developing struvite calculi}

Appropriate imaging tests are indicated in SCI patients who develop recurrent UTI in order to detect struvite calculi. In a retrospective review of 1669 patients with SCI between 1982 and 1996, the development of recurrent UTI was the most common mode of presentation of upper urinary tract struvite calculi. ${ }^{11}$

\section{SCI patients with hydronephrosis may present with} unconventional symptoms

The symptoms of hydronephrosis may be bizarre and non-specific in SCI patients. The symptoms include tiredness, not feeling well, abdominal discomfort, increased spasms, and autonomic dysreflexia. Persis- tent temperature indicates sepsis in an obstructed kidney. Physicians should be aware of the serious import of these symptoms in the individuals with SCI.

\section{In SCI patients, the urinary tract is often a source of sepsis}

The urinary tract should be suspected as a possible focus of sepsis in SCI patients manifesting symptoms of temperature, sweating, increased spasms, and recurrent autonomic dysreflexia, either alone or in any combination.

An obstructed kidney is susceptible to infection from a septic focus elsewhere in the body

When a SCI patient develops bacteremia from an infected, deep pressure sore, or from severe respiratory tract infection, an obstructed kidney is susceptible to a secondary infection. This is exemplified by the course of events in two tetraplegic patients. In the case of Patient $D$, the primary focus of infection was a pressure sore in the groin, which was extending to the hip joint. The obstructed right kidney became infected secondarily. Patient $\mathrm{C}$, who was a C-4 tetraplegic, developed severe chest infection and the obstructed kidney had a secondary infection. At post mortem, pyonephrosis with perinephric abscess was discovered.

\section{Perinephric xanthogranulomatous inflammation in a spinal cord injury patient}

Spinal cord injury patients with upper urinary tract calculi and recurrent UTI may develop xanthogranulomatous inflammation in the kidney and/or in the perinephric tissue. Post mortem in Patient $\mathrm{C}$ revealed pyonephrosis with an abscess between the left kidney and left hemi-diaphragm. Histology showed chronic pyelonephritis of the left kidney associated with perinephric abscess and xanthogranulomatous inflammation of perinephric fatty tissue.

\section{A delay in the diagnosis of hydronephrosis can lead to loss of renal function}

Belated diagnosis of hydronephrosis and diagnosis of obstructive uropathy after sepsis has supervened, often results in irrevocable loss of ipsilateral renal function. In Patient B, MAG-3 renogram showed no appreciable function in the obstructed kidney, although an IVU performed 3 years earlier showed normal left kidney.

Considerable renal function may be lost when sepsis supervenes in a hydronephrotic kidney

Unless prompt drainage is provided to an obstructed kidney after sepsis has supervened, there is often irrevocable loss of renal function. In Patient $\mathrm{D}$, who developed sepsis in the obstructed kidney, percutaneous nephrostomy was delayed. A follow-up MAG-3 
renogram showed a relative renal function of only $3 \%$. Therefore, urgent drainage is indicated in SCI patients who develop sepsis in a hydronephrotic kidney to achieve salvage of any residual renal function.

Percutaneous nephrostomy is indicated in SCI patients who develop sepsis in a hydronephrotic kidney

When a hydronephrotic kidney becomes infected in a SCI patient, percutaneous upper urinary tract drainage serves as a potentially life-saving measure. ${ }^{12,13}$ In patient A, percutaneous nephrostomy resulted in satisfactory drainage of the obstructed, infected kidney with subsidence of the features of sepsis. The physician performing percutaneous nephrostomy should be cognizant of the potential problems of percutaneous renal puncture in a SCI patient due to (i) spinal curvature; (ii) spasms; (iii) colonic distension due to faecal loading; and (iv) more medial and vertical positioning of the kidneys due to the psoas muscle atrophy. The lower pole of the right kidney was situated $98 \mathrm{~mm}$ from the midline in normal males; but in paraplegics, the distance was only $72.2 \mathrm{~mm}$ as the kidney assumes a more vertical position closer to the spine. ${ }^{14}$ Positioning for percutaneous nephrostomy may be difficult in a few SCI patients because of contractures at hip and knee joints. In Patient A, percutaneous access to the kidney for providing nephrostomy drainage was difficult. A CT scan performed after the percutaneous nephrostomy, revealed intrarenal and perirenal haematoma on the left side. There was mild psoas haematoma and thickening of the perirenal fascia (Figure 1C).

\section{Conclusions}

The urinary tract continues to be a prime cause of morbidity in many SCI patients. Evaluation and treatment of urinary tract ailments topped the list $(43.43 \%)$ for the reasons for readmission of tetraplegic patients to a spinal unit. $^{15}$ As any delay in the diagnosis of obstructive uropathy may lead to irreversible loss of renal function, beds should be available in the spinal unit for priority admission of the SCI patients presenting with urinary tract-related diseases. It is important that SCI patients are admitted to a spinal injuries unit so that they can obtain global care. This will ensure that prompt diagnosis is made, and comprehensive treatment is provided expeditiously for the SCI patients with hydronephrosis due to urinary calculi.

\section{References}

1 Strauther GR, Longo WE, Virgo KS, Johnson FE. Appendicitis in patients with previous spinal cord injury. Am J Surg 1999; 178: $403-405$.

2 Sheridan R. Diagnosis of the acute abdomen in the neurologically stable spinal cord-injured patient. A case study. J Clin Gastroent 1992; 15: 325-328.

3 Bar-On Z, Ohry A. The acute abdomen in spinal cord injury individuals. Paraplegia 1995; 33: 704-706.

4 Vaidyanathan $\mathrm{S}$ et al. Fatal intraperitoneal bladder rupture due to a blocked catheter in a paraplegic. Paraplegia 1979; 17: $272-$ 277.

5 Goswami AK, Suryaprakash B, Malik AK, Vaidyanathan S. Development of fatal bilateral xanthogranulomatous pyelonephritis in a paraplegic: case report. Paraplegia 1988; 26: 62-65.

6 Vaidyanathan $\mathrm{S}$ et al. Recurrent bilateral renal calculi in a tetraplegic patient. Spinal Cord 1998; 36: 454-462.

7 Waites KB et al. Compliance with annual urologic evaluations and preservation of renal function in persons with spinal cord injury. J Spinal Cord Med 1995; 18: $251-254$.

8 Canupp KC, Waites KB, DeVivo MJ, Richards JS. Predicting compliance with annual follow-up evaluations in persons with spinal cord injury. Spinal Cord 1997; 35: 314-319.

9 Vaidyanathan $\mathrm{S}$ et al. Letter to Editor re: Milk of calcium in the inferior calyx of a hydronephrotic kidney in a tetraplegic patient - a diagnosis to be made before scheduling for extracorporeal shock wave lithotripsy. Spinal Cord 38: 325-326.

10 Hammond MC, Britell CW, Little JW, DeLisa JA. Diagnostic ultrasound: its value in acute urinary tract infection in spinal cord injury. Arch Phys Med Rehabil 1987; 68: $743-744$.

11 Donnellan SM, Bolton DM. The impact of contemporary bladder management techniques on struvite calculi associated with spinal cord injury. Br J Urol 1999; 84: 280-285.

12 Watson RA et al. Percutaneous nephrostomy as adjunct management in advanced upper urinary tract infection. Urology 1999; 54: $234-239$.

13 Nocolescu D et al. Emergency percutaneous nephrostomy in the septic kidney. Acta Urologica Belgica 1992; 60: $27-32$.

14 Karasick D, Karasick S, Brennan R. Renal positional changes in spinal cord-injured patients. Am J Roentgen 1983; 140: 521 - 522 .

15 Vaidyanathan $\mathrm{S}$ et al. A review of the re-admissions of patients with tetraplegia to the Regional Spinal Injuries Centre, Southport, United Kingdom, between January 1994 and December 1995. Spinal Cord 1998; 36: $838-846$. 